\title{
CUTTING ERRORS IN
}

\section{TOTAL KNEE REPLACEMENT: ASSESSMENT BY COMPUTER ASSISTED SURGERY}

\begin{abstract}
$\underline{\text { Abstract }}$
The observed errors in the position of the implanted prosthesis can be due to a number of potential causes. One of these is the potential error during execution of the bone cuts. However, there is only minimal information on this in the current literature. The amount of cutting errors in forty consecutive total knee replacements was reported. All the operations were done by the same surgeon. The amount of cutting error was measured by the use of computer navigation system. It was hypothesized that there was no difference in the amount of error between bone cut through the cutting slot (slotted cutting) and bone cut done on the surface of the cutting guide (open cutting). It was found that the average absolute cutting error was $1^{\circ}$ in the coronal plane and $1.4^{\circ}$ in the sagittal plane. Significantly more outlier (more than $3^{\circ}$ ) was observed in the errors in the sagittal plane ( $\mathrm{p}=0.014$, chi square test). Open cutting resulted in less error in the sagittal plane of the tibial cut when compared with slotted cutting ( $\mathrm{p}=0.031$, Mann-Whitney U Test). This was attributed by the use of a thicker saw blade with higher stiffness in the open cutting method.
\end{abstract}

Keywords: Total Knee Replacement, Cutting error 


\section{$\underline{\text { Introduction }}$}

The success of total knee replacement depends on the correct alignment of the lower limb mechanical axis [ 5, 10 ]. It is recommended that the post-operative lower limb mechanical axis should be within three degrees from the neutral alignment. The tibial cut surface in the coronal plane should be made perpendicular to the mechanical axis of the tibia. A varus cutting error of more than three degrees in the tibial side was reported to be associated with decreased survivorship of the prosthesis [ 4 ].

Surgical outliers are not uncommon. Only around $70-80 \%$ of the reported alignment of the implanted total knee replacements was considered to be satisfactory [ $2-4,6-8,13-14$ ]. The percentage is only increased to around $90 \%$ even in the hands of expert [ 12 ].

The observed errors in the position of the implanted prosthesis can be due to a number of potential causes. One of these is the potential error during execution of the bone cuts. However, there is only minimal information on this in the current literature [ 1 , 9 ].

It is recommended by most of the instrumentation systems to have the bone cut carried out through the cutting slot of the cutting guide (slotted cutting). However, a relatively thinner saw blade has to be used. There is a concern about potential deflection of the saw blade at the far end, leading to an increased cutting error in the sagittal plane. Some surgeons prefer to perform the bone cut on the top of the cutting guide (open cutting). This allows the use of a thicker saw blade and minimizes the chance of saw blade deflection. 
Computer navigation assisted total knee replacement is introduced in recent years to improve the accuracy in the execution of bone cuts and implantation of prosthesis. Besides, it is also a unique tool in measuring the planes before and after the execution of bone cuts.

This paper reported the amount of errors in the execution of the bone cut in a series of conventional incision total knee replacement performed using computer navigation technique. The influence of slotted cutting versus open cutting on the accuracy of the bone cuts was discussed. 


\section{Method}

This was a retrospective review of a consecutive series of forty total knee replacements done under imageless computer navigation guidance. All the operations were done by the same surgeon (WPY) using standard operative technique. All the total knee replacements were performed by using a conventional incision technique. The usual length of incision ranged from $12 \mathrm{~cm}$ to $15 \mathrm{~cm}$. A tourniquet was inflated during the procedure. A standard medial para-patellar approach was used. The posterior cruciate ligament was sacrificed. The patella was everted laterally and the tibia was subluxed anteriorly. A tibial-cut-first policy was adopted. The distal femur cut was done after completion of the tibial cut. All the bone cuts were carried out in an anterior-posterior direction using the conventional cutting guides. The referencing landmark of the rotational alignment of the tibial cut was the tibial tuberosity. A routine posterior slope of 3 degrees was adopted in carrying out the tibial bone cut. The execution of the bone cuts were done either through the cutting slot (slotted cutting) or on the surface of the cutting guide (open cutting). The decision of slotted cutting versus open cutting was not randomized. A thin saw blade of a thickness of $0.89 \mathrm{~mm}$ (90 $\mathrm{mm}$ long and $25 \mathrm{~mm}$ wide) was used if the bone cut was carried out through the cutting slot. A thick saw blade of a thickness of $1.47 \mathrm{~mm}$ (90 mm long and $25 \mathrm{~mm}$ wide) was used if the bone cut was done on the surface of the cutting guide. The precision of the first-cut was verified using the computer navigation system. Repeated bone cuts were performed if the error of the first-cut was more than one degree from the planned alignment.

The amount of the first-cut errors with reference to the planned cutting plane in both the coronal plane and sagittal plane was measured by computer navigation system. 
40 consecutive total knee replacements were done in 28 patients. 7 were male and 21 were female. The average age of the patient was 69.5 years ( $\mathrm{SD}=9.6$ years). 16 patients received unilateral total knee replacements while 12 patients received one stage sequential bilateral total knee replacements. 36 knees were operated because of a diagnosis of primary osteoarthritis and 4 knees were operated because of rheumatoid arthritis.

It was hypothesized that there was no difference in the absolute errors of the bone cut between slotted cutting and open cutting. The null hypothesis was examined using Mann-Whitney U test. Statistical significance was assumed if $\mathrm{p}$ value was less than 0.05 


\section{$\underline{\text { Results }}$}

All the total knee replacements were performed using passive optical imageless computer navigation system designed by BrainLAB (Munich, Germany). The result of 80 bone cuts (40 tibial cuts and 40 distal femur cuts) was reported.

The average absolute first-cut cutting error of the tibial cut was 1.3 degrees in the coronal plane (ranged from valgus 2.5 degrees to varus 3 degrees) and 1.3 degrees in the sagittal plane (ranged from extension error of 4 degrees to flexion error of 1 degree). The average absolute first-cut cutting error of the distal femur cut was 0.8 degree in the coronal plane (ranged from valgus 2 degrees to varus 2.5 degrees) and 1.6 degrees in the sagittal plane (ranged from extension 3.6 degrees to flexion 5.6 degrees) (Figure 1).

If an error in the bone cut of greater than 3 degrees were considered as surgical outlier, no outlier was found in the coronal plane (both tibial cut and distal femur cut). Concerning the error of the bone cuts in the sagittal plane, one tibial cut and five distal femur cuts had an error of greater than 3 degrees. Significantly more outliers were found in the sagittal plane than the coronal plane ( $\mathrm{p}=0.014$, chi square test).

58 cuts (28 tibial cuts and 30 distal femur cuts) were done through the cutting slot and 22 cuts (12 tibial cuts and 10 distal femur cuts) were done on the surface of the cutting guide (Table 1). Significantly more error in the sagittal cutting plane of the tibial cut was found when the bone cut was done through the cutting slot (absolute error in slotted cutting $=1.5^{\circ}+/-0.8^{\circ} ;$ absolute error in open cutting $=0.9^{\circ}+/-0.9^{\circ}$ ) ( $\mathrm{p}=0.031$, Mann-Whitney U Test). 


\section{$\underline{\text { Discussion }}$}

The margin of error of the optimal implant alignment in total knee replacement is narrow. A varus cutting error of more than three degrees in the tibial side was reported to be associated with decreased survivorship of the prosthesis [ 11 ]. Error in the execution of bone cuts is one of the major potential errors leading to malalignment of the implanted total knee prosthesis. It is reported in one cadaveric study that the maximum cutting errors relative to the cutting guide are in the range of $1.5^{\circ}$ to $2^{\circ}$ (varus-valgus) and $3^{\circ}$ to $4^{\circ}$ (flexion-extension) [ 9 ].

The average absolute cutting error reported in the current study ranged from 0.8 degree (distal femur cut) to 1.3 degrees (tibia cut) in the coronal plane (i.e. varusvalgus error) and 1.3 degrees (tibial cut) to 1.6 degrees (distal femur cut) in the sagittal plane (flexion-extension error). The observed cutting error was higher in the sagittal plane when compared with the coronal plane. This echoed the results of similar studies done in cadaveric setting [ 9 ] and clinical setting [ 1 ].

All the bone cuts in the current study were done in the conventional anterior-posterior direction. The reason of the higher error in the sagittal plane was likely related to the potential deflection of the far end of the saw blade, especially when a thin saw blade was used. This led to an increase in flexion-extension error in the sagittal plane, as opposed to the varus-valgus error in the coronal plane. In order to minimize the potential saw blade deflection, it is reasonable to suggest the use of a thicker saw blade with higher stiffness whenever possible. 
It was observed in the current study that slotted cutting resulted in significantly higher error in the sagittal plane of the tibial cut when compared with open cutting $(\mathrm{p}=0.031$, Mann-Whitney U Test). In this study, a thicker saw blade of $1.47 \mathrm{~mm}$ was used for open cutting while a thinner saw blade of $0.89 \mathrm{~mm}$ was used for slotted cutting. We believed that the stiffness of the saw blade was the key factor explaining the difference observed between slotted cutting and open cutting. This observation differed from the reported findings in one cadaveric experiment, in which the same thickness saw blade (1.19-mm-thick) was used for both slotted cutting and open cutting [ 9 ]. However, the limitations of the current report in terms of the retrospective nature of the study and the limited number of observer involved suggested extreme caution in interpreting these results. Besides, there are other slotted cutting blocks in the market which accept a saw blade whose thickness is more than $1 \mathrm{~mm}$. It may not be appropriate to extrapolate the results of the current experiment to total knee replacements performed using those slotted cutting blocks. Readers of the journal should be aware of this.

Before the era of computer navigation orthopaedic surgery, attempts in measuring the potential errors in each step of total knee replacement (including the intra-operative bone cutting errors) were often cumbersome. On the other hand, the extrapolation of results coming from cadaveric experiment into clinical practice always raises a concern on the reproducibility in the in vivo situation. The use of computer navigation technique during total knee replacement allows convenient measurement of the potential errors intra-operatively (including the errors coming from anatomical variation of the bones, errors in the bone cut, errors in the implantation of the prosthesis, etc). This helps to improve the understanding of the magnitude and the relative importance of errors in each step of the operation. 
To conclude, the intra-operative first-cut cutting errors in forty consecutive total knee replacements were reported. The average absolute error in the sagittal plane (1.4 degrees) was higher than that in the coronal plane (1 degree). Significantly more outlier was observed in the errors in the sagittal plane ( $\mathrm{p}=0.014$, chi square test). Open cutting resulted in less error in the sagittal plane of the tibial cut when compared with slotted cutting ( $\mathrm{p}=0.031$, Mann-Whitney U Test). This was attributed by the use of a thicker saw blade with higher stiffness in the open cutting method. 


\section{Reference}

1. Bathis H, Perlick L, Tingart M, etc: Intraoperative cutting errors in total knee arthroplasty. Arch Orthop and Trauma Surg 125:16-20, 2005.

2. Bono JV, Roger DJ, Laskin RS, Peterson MG and Paulsen CA: Tibial intramedullary alignment in total knee arthroplasty. American J Knee Surg 8: 711, 1995.

3. Brys DA, Lombardi Jr AV, Mallory TH, Vaughn BK: A comparison of intramedullary and extramedullary alignment systems for tibial component placement in total knee arthroplasty. Clin Orthop 263:175-179, 1991.

4. Elloy MA, Manning MP and Johnson R: Accuracy of intramedullary alignment in total knee replacement. J Biomedical Engineering 14: 363-70, 1992.

5. Insall JN, Binazzi R, Soudry M and Mestriner LA: Total knee arthroplasty. Clin Orthop 192:13-22, 1985.

6. Jeffery RS, Morris RW and Denham RA: Coronal alignment after total knee replacement. J Bone Joint Surg Br 73-B: 709-14, 1991.

7. Ko PS, Tio MK, Ban CM, Mak YK, Ip FK, Lam JJ: Radiologic analysis of the tibial intramedullary canal in Chinese varus knees. Implications in total knee arthroplasty. J Arthroplasty 16:212-215, 2001. 
8. Maestro A, Harwin SF, Sandoval MG, Vaquero DH and Murcia A: Influence of intramedullary versus extramedullary guides on final total knee arthroplasty component position: a radiographic analysis. J Arthroplasty 13: 552-8, 1998.

9. Plaskos C, Hodgson AJ, Inkpen K and McGraw RW: Bone Cutting Errors in Total Knee Arthroplasty. J Arthroplasty 17:698-705, 2002.

10. Rand JA and Coventry MB: Ten-year evaluation of geometric total knee arthroplasty. Clin Orthop 232:168-73, 1988.

11. Ritter MA, Faris PM, Keating EM and Meding JB: Postoperative alignment of total knee replacement. Its effect on survival. Clin Orthop 299:153-6, 1994.

12. Stulberg SD. Loan P and Sarin V: Computer-assisted navigation in total knee replacement: results of an initial experience in thirty-five patients. J Bone Joint Surg Am 84-A Supp 2:90-8, 2002.

13. Teter KE, Bregman D and Colwell CWJ: Accuracy of intramedullary versus extramedullary tibial alignment cutting systems in total knee arthroplasty. Clin Orthop 321: 106-10, 1995.

14. Yang SH and Liu TK: Intramedullary versus extramedullary tibial alignment guides in total knee arthroplasty. J Formosan Medical Association 97: 564-8, 1998. 


\section{Legend of Figures}

Figure 1 First-Cut Cutting errors of Tibia Cut and Distal Femur Cut (Both

Coronal Plane and Sagittal Plane)
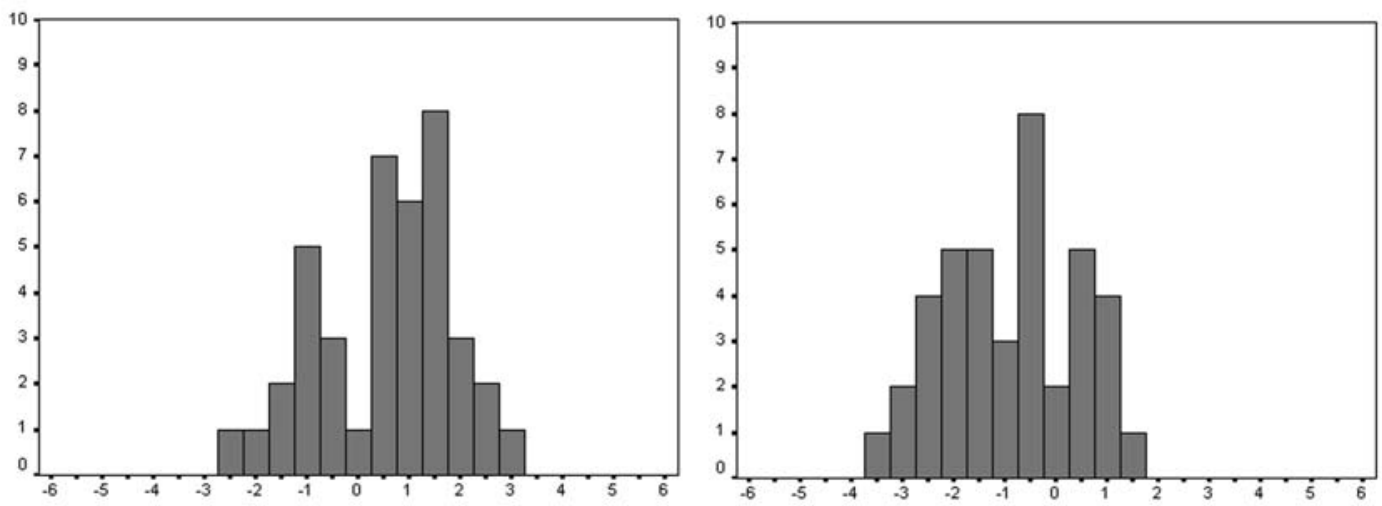

Tibial Cut Coronal Plane Cutting Error (Varus: +ve; Valgus:-ve) (Deg)

Tibial Cut Saqittal Plane Cuttina Error (Flex+ve: Ext-ve) (Dea)
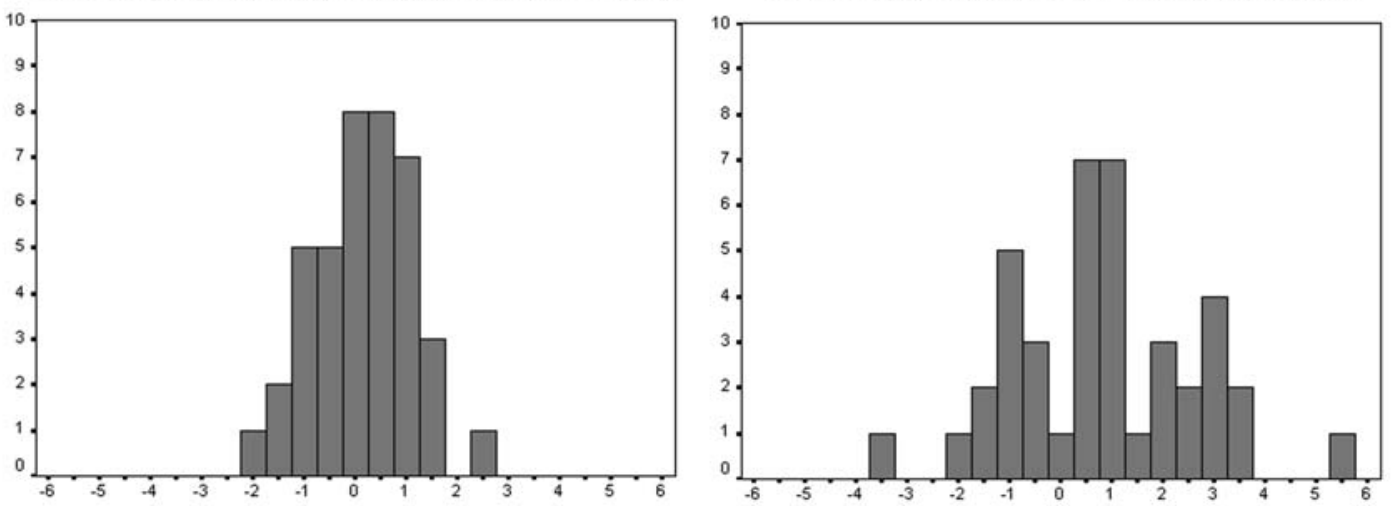

Distal Femur Coronal Plane Cutting Error (Varus:+ve; Valgus:-ve) (Deg

Distal Femur Sagittal Plane Cutting Error (Flex+ve; Ext-ve) (Deg) 